\title{
The Eighth Edition of TNM: Implications for the Surgical Oncologist
}

\author{
David R. Byrd, MD, FACS ${ }^{1}$ and Frederick L. Greene, MD, FACS ${ }^{2}$ \\ ${ }^{1}$ Department of Surgery, University of Washington School of Medicine, Seattle, WA; ${ }^{2}$ Levine Cancer Institute, Charlotte, \\ NC
}

Since 1977, the American Joint Committee on Cancer (AJCC) has published eight editions of cancer staging manuals using contemporary evidenced-based literature to build a common language of cancer for the care of cancer patients by clinicians and for the cancer surveillance community. The interval between these editions, approximately 5-7 years, has been necessary to balance the publication of a critical number of important changes in knowledge of cancer with a need for longitudinal comparison of cancer incidence, prognosis, and survival. There were a number of new chapters and changes in the 7th edition of the AJCC Cancer Staging Manual that were published in an overview editorial from $2010 .{ }^{1}$ In the 8th edition, updated information regarding new and clinically relevant prognostic molecular markers has been added to traditional anatomical staging to ensure that the clinical and pathological classification of cancer will remain relevant to the surgical oncology community and to those who have oversight for cancer surveillance. ${ }^{2}$

The extent or stage of cancer at the time of diagnosis is a key factor that defines prognosis and is a critical element in determining appropriate treatment based on the experience and outcomes of groups of previous patients with similar stage. The core of the language of solid tumors has been $\mathrm{T}$ (tumor) $\mathrm{N}$ (node) $\mathrm{M}$ (metastasis) staging introduced by a surgeon, Pierre Denoix, in the mid-twentieth century and enhanced by the Union for International Cancer Control (UICC) and the AJCC over the next 7 decades. ${ }^{3}$ Complete or near-complete TNM element collection is available in

\section{(C) Society of Surgical Oncology 2017}

First Received: 5 July 2017;

Published Online: 7 August 2017

F. L. Greene, MD, FACS

e-mail: fgreene@med.unc.edu countries worldwide and has provided a backbone of anatomical staging to compare the incidence, extent of disease, and prognosis across continents. Both the AJCC and UICC are committed to updating and collecting these TNM elements for anatomical stage groupings. However, in high-, many middle-, and some low-income countries, additional prognostic and biological factors are routinely collected and used to counsel and treat patients. In the management of breast cancer patients, for example, the status of tumor grade, estrogen and progesterone receptor positivity, and the presence of HER2/neu gene overexpression often supersede the TNM tumor status for systemic and even locoregional treatment recommendations.

The challenges that have become increasingly evident since 2010 include: (1) the need and adoption of nonanatomical prognostic factors based on the biological behavior of cancers in addition to the TNM extent of disease to identify and counsel patients on prognosis and treatment options; (2) the limitations in the levels of evidence used in many widely accepted publications on cancer; (3) the need for incorporating anatomical and functional imaging into staging; (4) the need to collect and incorporate staging data into electronic health systems and other shared databases; and (5) the virtual tsunami of new and existing biological data elements that are being discovered and collected with exponential speed that now require a systems biology approach and computational bioinformatics analysis to bring sense and meaning to our understanding of cancer (Fig. 1).

The 8th edition of the AJCC Cancer Staging Manual, introduced in late 2016, will be required for staging patients beginning January 1, 2018. To benefit from a greater cross-section of cancer experts, in addition to the involvement of site-specific expert clinician panels, new Core Groups were introduced: precision medicine, 


\section{AJCC Vision}

The Transition from Population Based to a more

"Personalized" Approach

Cancer Stage

\section{Comprehensive Cancer Profile}

AJCC/UICC TNM Stage

(Basic Classification)

AJCC Prognostic Stage Groups

(Advanced Clinical Relevance)

Prognostic

Factors

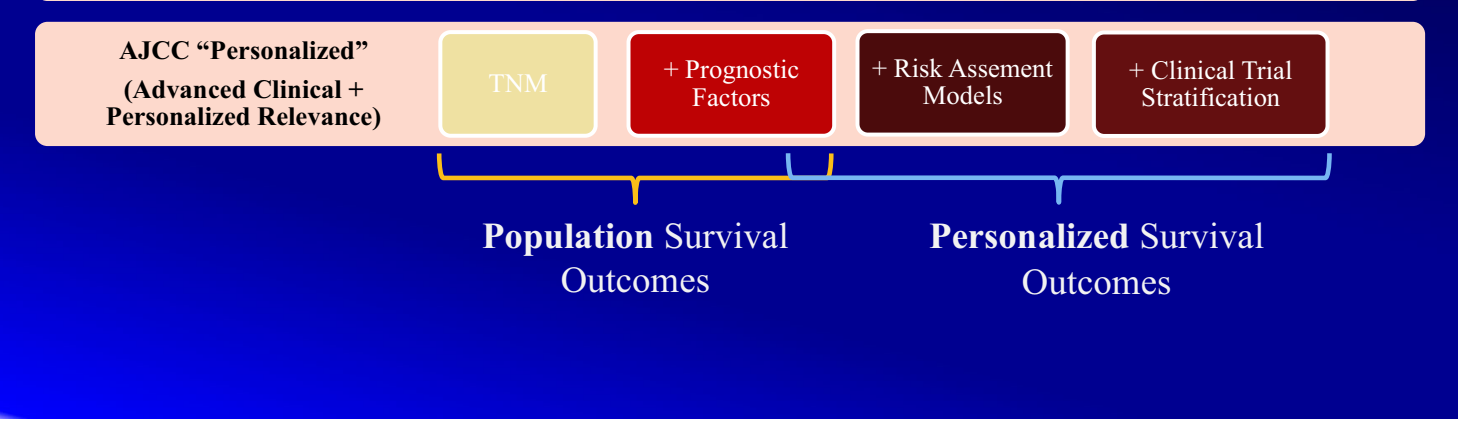

FIG. 1 The vision of the 8th edition, AJCC Cancer Staging Manual

evidence-based medicine and statistics, imaging, content harmonization, and data collection cores. Eighteen expert panels (formerly known as task forces) include contributors from 181 institutions, 22 countries, and 6 continents, all as volunteer content experts. In Chap. 1, "Principles of Cancer Staging," the rules of TNM staging are fully described and include results of a TNM "harmonization summit" conducted by the AJCC that also included key representatives from the UICC. New paradigms and new features were added to the 8th edition to incorporate new knowledge of tumor biology and updated clinical strategies for cancer treatment (Table 1). Many new chapters were created and one was deleted in the 8th edition (Table 2). Several chapters were split or merged in the 8th edition (Table 3).

In addition to completing an electronic version of the Manual, the AJCC has developed a Content Component Management System (CCMS) and application programming interface (API) that will allow ongoing updates and revisions that can be incorporated into electronic health systems, tumor registries, centralized national cancer databases, and other cancer stakeholders' systems that will ensure the fidelity of cancer data collection and incorporation into cancer staging. AJCC's vision for future content
TABLE 1 New paradigms and new features

\section{New paradigms}

Separation of oropharyngeal carcinoma staging based on human papilloma virus (HPV) status

Separation of staging systems for patients receiving neoadjuvant systemic treatment in selected sites (esophagus and stomach)

Separate staging systems for bone and soft tissue sarcoma base on anatomical site

\section{New features}

Provision of levels of evidence (LOE) when revisions to staging systems are made

Inclusion of risk assessment models for selected cancer sites

Subdivision of prognostic factors into: 1. required for staging, 2. recommended for clinical care, and 3. emerging factors

and products includes the development of prognostic staging calculators and an ongoing commitment of the validation of risk assessment tools and nomograms and inclusion criteria for stratification into clinical trials. For example, the development and use of staging calculators will be essential to stage breast cancer patients where several hundred tables need to be reviewed to calculate the prognostic stage group when all possible permutations that 
TABLE 2 New and deleted chapters

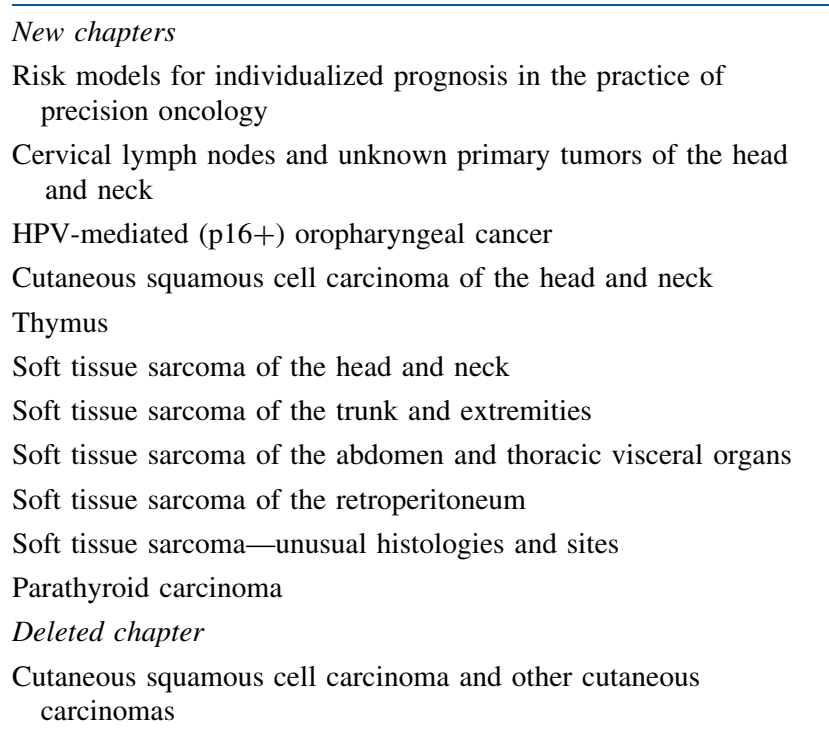

TABLE 3 Split and merged chapters

Split chapters
Oropharynx (p16-) and hypopharynx
Nasopharynx
Exocrine pancreas
Neuroendocrine tumors of the pancreas
Neuroendocrine tumors of the stomach
Neuroendocrine tumors of the duodenum and ampulla of vater
Neuroendocrine tumors of the jejunum and ileum
Neuroendocrine tumors of the appendix
Neuroendocrine tumors of the colon and rectum
Thyroid - differentiated and anaplastic carcinoma
Thyroid-medullary
Adrenal cortical carcinoma
Adrenal-neuroendocrine
Merged chapters
Ovary, fallopian tube, and primary peritoneal carcinoma

include grade, estrogen and progesterone status, and HER2 overexpression, prognostic gene panels, and $\mathrm{T}, \mathrm{N}$, and $\mathrm{M}$ categories are taken into account. These eight factors are now required for prognostic stage grouping when available. In addition to these much needed additions, the Breast Expert Panel, along with the Editorial Board of the 8th edition, has now recommended that lobular carcinoma in situ (LCIS), including the pleomorphic variant, should no longer be considered a cancer and, therefore, should not be staged using TNM.

One ongoing tradition followed in each edition of the AJCC Cancer Staging Manual is to dedicate the compendium of work to an individual who has contributed significantly to the concepts espoused by the AJCC. The Editorial Board of the 8th edition departed from this tradition by dedicating the Manual to our Cancer Registrars who work tirelessly to codify the data that are used not only in staging but also in the support of clinical research and especially clinical trials. The Cancer Registrars have been recognized in addition for their "professionalism in the collection of factors that are fundamental to sustaining local, state and national cancer registries" and for their "leadership, support and promulgation of the principles of cancer staging and their positive impact on patient cancer outcomes." We could think of no more meaningful dedication!

The overarching principles found throughout the 8th edition support the concept of a team approach to cancer staging by clinical oncologists which includes cancer registrars, pathologists, and radiologists. The assignment of cancer stage is ultimately the responsibility of the managing cancer providers-surgeons, medical oncologists, radiation oncologists-who have access to the complete data set. Leading up to January 1, 2018, the expectation is that all clinicians who provide cancer care will continue to document 7 th edition cancer staging while also becoming familiar with the $8^{\text {th }}$ edition content to aid in counseling their patients on the current best evidence for prognosis and treatment. We believe that familiarity with specific disease site chapter content and Chap. 1, "Principles of Cancer Staging," will be essential to stage patients accurately and to utilize our "language of cancer" correctly.

\section{REFERENCES}

1. Edge SB, Compton CC. The American Joint Committee on cancer: the 7th edition of the AJCC Cancer Staging Manual and the future of TNM. Ann Surg Oncol. 2010;17:1471-4.

2. Amin MB, Edge SB, Greene FL, et al. (eds) AJCC cancer staging manual, 8th ed. Springer: New York; 2017.

3. Denoix PF. Nomenclature classification des cancer. Bull Inst Nat Hyg (Paris). 1952;7:743-8. 